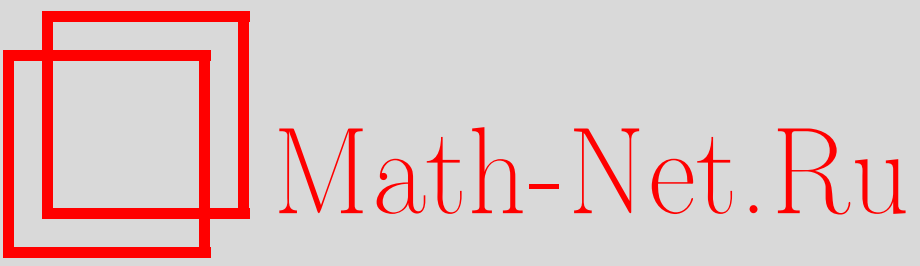

Е. К. Алексеев, Л. Р. Ахметзянова, И. Б. Ошкин, С. В. Смышляев, Обзор уязвимостей некоторых протоколов выработки общего ключа с аутентификацией на основе пароля и принципы построения протокола SESPAKE, Mameм. вопр. криптогр., 2016, том 7, выпуск 4, 7-28

DOI: https://doi.org/10.4213/mvk201

Использование Общероссийского математического портала Math-Net.Ru подразумевает, что вы прочитали и согласны с пользовательским соглашением

http: //www.mathnet.ru/rus/agreement

Параметры загрузки:

IP : 3.95 .254 .165

26 апреля 2023 г., 15:36:39 
МАТЕМАТИЧЕСКИЕ ВОПРОСЫ КРИПТОГРАФИИ

2016 T. 7 № 4 C. $7-28$

УДК 519.719.2

\title{
Обзор уязвимостей некоторых протоколов выработки общего ключа с аутентификацией на основе пароля и принципы построения протокола SESPAKE
}

\author{
Е. К. Алексеев, Л. Р. Ахметзянова, И. Б. Ошкин, С. В. Смышляев
}

ООО «КРИПТО-ПРО», Москва

Получено 23.VI.2016

\begin{abstract}
Аннотация. Рассматривается семейство протоколов, позволяющих сторонам, имеющим малоэнтропийный общий секрет (пароль), выработать высокоэнтропийный общий ключ, взаимодействуя по незащищенному каналу. Предполагается, что противник может проверить истинность предполагаемого пароля, только вступив в активное взаимодействие с одной из сторон. Приводится обзор известных уязвимостей протоколов этого семейства. На их примерах объясняются основные принципы построения российского протокола SESPAKE.

Работа выполнена при поддержке Российского фонда фундаментальных исследований, проект 16-01-00226 А.

Ключевые слова: протоколы, SESPAKE, PAKE, выработка ключа, аутентификация, пароль
\end{abstract}

A review of the password authenticated key exchange protocols vulnerabilities and principles of the $S E S P A K E$ protocol construction

E. K. Alekseev, L. R. Akhmetzyanova, I. B. Oshkin, S. V. Smyshlyaev

CryptoPro LLC, Moscow

Abstract. We consider a family of protocols permitting the parties sharing a weak key (a password) to generate a strong common key using the unprotected channel. It is supposed that to check the password correctness the adversary should interact with legitimate participants. A review of the known vulnerabilities of the protocols of the family considered is given. This review is used to explain the main principles of the SESPAKE protocol construction.

Key words: protocols, SESPAKE, PAKE, key exchange, authentication, password Citation: Mathematical Aspects of Cryptography, 2016, v. 7, № 4, pp. 7-28 (Russian)

(c) Академия криптографии Российской Федерации, 2016 г. 


\section{1. Введение}

Существует множество протоколов аутентифицированной выработки общего ключа. Некоторые из них, например STS [6] или протокол НидхемаШредера [7], предполагают использование сертификатов или, в общем случае, наличие доверенной третьей стороны. Другие, например, AKEP2 [5] или TLS-PSK [4], основаны на том, что взаимодействующие стороны изначально владеют некоторым общим секретом. В последнем случае возникает проблема безопасного хранения разделяемого сторонами общего секрета. Есть несколько путей решения этой проблемы, один из которых заключается в простом запоминании секрета пользователем системы. С практической точки зрения данный подход является реалистичным лишь тогда, когда секрет можно запомнить сравнительно легко. Это возможно, например, в случае использования паролей. Однако пароли являются так называемыми малоэнтропийными секретами - даже если пароль имеет большую длину, он в большинстве случаев выбирается в соответствии с распределением, далеким от равномерного. По этой причине становится возможным осуществление так называемых «словарных атак», заключающихся в переборе паролей от высоковероятных к маловероятным. Данный подход зачастую является существенно более эффективным по сравнению с полным перебором, а восстановление даже длинного пароля оказывается осуществимым на практике. В том же случае, когда пароль является априори коротким (как, например, ПИН-код от банковской карты), ни о какой устойчивости даже к обычной переборной атаке говорить не приходится. Данные атаки могут быть реализованы, если после прослушивания процесса взаимодействия сторон или активного вмешательства в это взаимодействие противник получает некоторую информацию, позволяющую ему проверить истинность своего предположения о пароле. Так, прослушав взаимодействие сторон по протоколу $A K E P 2$, противник получает критерий для подбора любого из двух разделяемых сторонами ключей. Поэтому необходимым условием стойкости протокола $A K E P 2$ является использование высокоэнтропийных ключей - ключей, выбранных из большого множества в соответствии с равномерным распределением.

Приведенные выше рассуждения показывают, что при использовании пароля в качестве общего секрета должны разрабатываться специальные протоколы. Необходимость применения именно таких протоколов может возникать, например, в случае хранения ключа пользователя в ключевом хранилище («токене»), взаимодействие с которым осуществляется по каналу связи, допускающему присутствие в нем активного противника. В этом случае у пользователя нет иных вариантов, кроме как использовать пароль для доступа к токену (иначе ему пришлось бы снова решать задачу безопасного хранения ключа доступа к этому токену). 
В 1992 году в работе [9] Стивеном Белловином и Майклом Мерритом был предложен первый протокол выработки общего ключа с аутентификацией на основе пароля. Впоследствии он стал прототипом для целого семейства протоколов, известного под названием PAKE («Password-Authenticated Key Exchange»). Суть протоколов PAKE состоит в том, что стороны, изначально разделяя лишь малоэнтропийный ключ (пароль), в процессе взаимодействия вырабатывают высокоэнтропийный общий сессионный ключ. При этом даже активный противник, имеющий доступ к каналу связи, не может получить информацию, достаточную для подбора пароля «offline» - то есть без взаимодействия с легитимными участниками.

Одно из первых формальных описаний модели противника для протоколов этого семейства появилось в 2000 году в [8]. А в 2005 году в [1] был построен протокол выработки общего ключа на основе пароля, который лег в основу этапа выработки ключа протокола SESPAKE («Security Evaluated Standardized Password Authenticated Key Exchange»), разработанного российскими специалистами и принятого в России в 2015 году в качестве методических рекомендаций ТК26 (Технический комитет по стандартизации «Криптографическая защита информации»).

В данной статье рассмотрены уязвимости ряда протоколов, в той или иной степени схожих с SESPAKE по строению и применяемым низкоуровневым механизмам. Также описываются методы, эксплуатирующие эти уязвимости для реализации различных угроз. При этом рассматриваются как реально существующие протоколы (при публикации которых авторами предполагалась их стойкость), так и «игрушечные», то есть созданные специально для демонстрации важности учета каких-то конкретных принципов. В заключительной части работы описанные уязвимости используются для того, чтобы объяснить конструктивные особенности протокола SESPAKE.

\section{2. Обозначения и основные определения}

Будем использовать обозначение $\mathbb{G}$ для произвольной мультипликативной циклической группы простого порядка $q$, через $g$ и $h$ будем обозначать её образующие элементы, а через $1_{\mathbb{G}}-$ нейтральный элемент группы $\mathbb{G}$.

Через $\mathbb{E}$ будем обозначать подгруппу простого порядка $q$ группы точек некоторой эллиптической кривой порядка $m$. При этом $P$ - порождающий элемент подгруппы $\mathbb{E}$, а $0_{\mathbb{E}}-$ ее нейтральный элемент.

Через $P W$ будем обозначать пароль, случайно выбранный из множества паролей $\mathcal{D}$. Запись $a \in \mathcal{U} A$ означает, что величина $а$ является реализацией случайной величины, равномерно распределенной на множестве $A$. Пусть $H$ - хэш-функция, $\mathcal{E}_{K}-$ блочный шифр, где $K-$ ключ. Через $\mathcal{E}_{K}^{-1}$ будем обозначать функцию, обратную $\mathcal{E}_{K}$. 
Пусть $M A C_{K}$ - функция генерации кода аутентификации сообщения, зависящая от ключа $K$. Через $F$ будем обозначать функцию, которая каждому элементу из множества $\mathcal{D}$ ставит в соответствие элемент из группы $\mathbb{G}$ или $\mathbb{E}$ (из какой конкретно, всегда ясно из контекста).

Всюду далее в схемах протоколов через $I D_{A}$ и $I D_{B}$ будем обозначать идентификаторы участников протокола $A$ и $B$ соответственно. Если протокол несимметричный, под стороной $A$ обычно подразумевается клиент, а под стороной $B$ - сервер. Через $K_{A}, K_{B}$ будем обозначать сессионные ключи, выработанные в ходе выполнения протокола на стороне $A$ и на стороне $B$. Также через $T_{A}$ и $T_{B}$ будем обозначать значения, вычисленные на этапе подтверждения ключа соответствующими сторонами. Далее для краткости описания протоколов будем предполагать, что если условия принадлежности элемента множеству или равенства значений не выполнены, то сторона, осуществляющая их проверку, завершает выполнение протокола с ошибкой.

\section{3. Модели противника для протоколов РAKE}

Исследование стойкости протокола в первую очередь предполагает выбор модели противника, а именно определение возможностей противника и определение угрозы - задачи по нарушению конфиденциальности или аутентичности, стоящей перед противником. В данном разделе приведено не формальное математическое описание модели противника, а лишь описаны основные идеи, лежащие в основе корректного и полного определения стойкости протоколов выработки общего ключа с аутентификацией на основе пароля.

Основными угрозами для протоколов семейства $P A K E$ являются полное или частичное вскрытие пароля или сессионного ключа. Легко видеть, что компрометация пароля приводит к незащищенности всех последующих соединений при наличии активного противника в канале.

Рассмотрим более подробно угрозу вскрытия пароля. Изначально предполагается, что пароль является малоэнтропийной величиной, а следовательно, его определение путем полного перебора (или по словарю) осуществимо на практике при наличии возможности эффективно проверять истинность опробуемого пароля. Априори противник может вступить во взаимодействие с одной из сторон и попытаться установить соединение с помощью предполагаемого им пароля. Если соединение установить удалось, то противник делает вывод о том, что предполагаемый им пароль совпадает с истинным. Такой способ проверки называется online-опробованием, а последовательная проверка всех возможных паролей таким образом - online-перебором. Методы противодействия online-перебору относятся скорее к техническим вопросам эксплуатации протокола и обсуждаются в разделе 5.3. 
Другим (уже не всегда доступным противнику) методом проверки паролей является проверка некоторого соотношения, зависящего от пароля, без активного взаимодействия с кем-либо. При этом такое соотношение должно допускать эффективную проверку. Последовательное опробование паролей таким способом называется offline-перебором.

Возможность осуществления offline-перебора определяется конструктивными особенностями протокола, а наличие такой возможности не позволяет говорить о его стойкости. Везде далее угроза вскрытия пароля и угроза получения противником информации, достаточной для осуществления offlineперебора, отождествляются.

Другая угроза для протоколов PAKE связана с этапом подтверждения ключа.

Большинство протоколов семейства $P A K E$ содержит этот этап - на нем стороны удостоверяются, что выработали один и тот же сессионный ключ. Для таких протоколов отдельно выделяется угроза, состоящая в том, что противнику удается убедить хотя бы одну из сторон, что другая сторона выработала тот же сессионный ключ. Данную угрозу будем называть угрозой ложной аутентификации, так как в случае ее реализации нарушается свойство аутентификации сессионного ключа.

Перейдем к определению возможностей противника по взаимодействию с системой.

Прежде всего предполагается, что все открытые параметры протокола противнику известны. Стойкость протоколов выработки общего ключа рассматривается относительно активного противника в канале, способного перехватывать и изменять сообщения, а также первым инициировать соединение с любой легитимной стороной.

Обычно модель противника предполагает, что в одно и то же время только один сеанс протокола между двумя участниками может быть незавершенным. Однако иногда рассматриваются модели, в которых несколько сеансов протокола могут быть активными одновременно.

Выработанный в протоколе $P A K E$ сессионный ключ зачастую продолжает использоваться при взаимодействии по протоколам более высокого уровня. Поэтому в модели необходимо учитывать возможность противника узнать этот ключ уже после завершения взаимодействия в соответствии с анализируемым протоколом семейства $P A K E$. Рассмотрение такой возможности обосновывается тем, что секретность ключа может быть нарушена в случае его использования в уязвимых высокоуровневых протоколах (например, из-за использования в них «слабых» шифров). 


\section{4. Уязвимости и принципы построения протоколов семейства $P A K E$}

Один из подходов к проектированию и исследованию протоколов предполагает анализ уязвимостей уже существующих протоколов подобного типа и методов, эксплуатирующих эти уязвимости для реализации различных угроз.

В данном разделе рассмотрены атаки как на существующие протоколы семейства $P A K E$, так и на «игрушечные» схемы. С помощью этих примеров подчеркиваются основные особенности протоколов семейства $P A K E$ и демонстрируется важность следования определенным принципам при построении отдельных этапов протокола: выбора открытых параметров, этапа выработки сессионного ключа и этапа его подтверждения.

\section{1. Неявная аутентификация ключа}

В данном разделе рассматривается связь такого свойства, как неявная аутентификация ключа (означающего, что взаимодействующие по протоколу стороны могут выработать общий ключ только в том случае, если разделяют один и тот же пароль), с основным требованием к протоколам семейства PAKE отсутствием у активного противника в канале возможности получить информацию, достаточную для осуществления offline-перебора паролей. В качестве первого примера рассмотрим «игрушечную» схему протокола (табл. 1).

Таблица 1. Уязвимая схема протокола семейства $P A K E$

\begin{tabular}{|c|c|c|}
\hline \multicolumn{3}{|c|}{ Открытые параметры: $\mathbb{G}, q, g, \mathcal{D}$} \\
\hline$A: I D_{A}, P W$ & & $B: I D_{B}, P W$ \\
\hline $\begin{array}{c}x_{A} \in \mathcal{U}\{1, \ldots, q-1\} \\
X_{A}=g^{x_{A}} \\
X_{B} \stackrel{?}{\in} \mathbb{G} \backslash\left\{1_{\mathbb{G}}\right\} \\
K_{A}=H\left(X_{B}^{x_{A}} \| P W\right)\end{array}$ & $\underset{\stackrel{I D_{A}, X_{A}}{\longrightarrow}}{\stackrel{I}{\longrightarrow}}$ & $\begin{array}{c}x_{B} \in \mathcal{U}\{1, \ldots, q-1\} \\
X_{B}=g^{x_{B}} \\
X_{A} \stackrel{?}{\in} \mathbb{G} \backslash\left\{1_{\mathbb{G}}\right\} \\
K_{B}=H\left(X_{A}^{x_{B}} \| P W\right)\end{array}$ \\
\hline $\begin{array}{l}T_{A}=H\left(I D_{A}\left\|I D_{B}\right\| X_{A}\left\|X_{B}\right\| K_{A} \| 1\right) \\
T_{B} \stackrel{?}{=} H\left(I D_{A}\left\|I D_{B}\right\| X_{A}\left\|X_{B}\right\| K_{A} \| 2\right)\end{array}$ & $\stackrel{T_{A}}{\longrightarrow}$ & $\begin{array}{l}T_{A} \stackrel{?}{=} H\left(I D_{A}\left\|I D_{B}\right\| X_{A}\left\|X_{B}\right\| K_{B} \| 1\right) \\
T_{B}=H\left(I D_{A}\left\|I D_{B}\right\| X_{A}\left\|X_{B}\right\| K_{B} \| 2\right)\end{array}$ \\
\hline
\end{tabular}


Такой протокол является уязвимым относительно угрозы вскрытия пароля в случае присутствия активного противника в канале. Противник подменяет собой сторону $B$, взаимодействуя с легитимным участником $A$ :

1. Легитимная сторона $A$ пересылает противнику сообщение $I D_{A}, X_{A}$.

2. Противник отправляет $A$ сообщение $I D_{B}, X_{E}$, где $X_{E}=g^{x_{E}}, x_{E} \in$ $\{1, \ldots, q-1\}$.

3. На легитимной стороне $A$ вырабатывается ключ $K_{A}=H\left(X_{A}^{x_{E}} \| P W\right)$. Заметим, что значение $X_{A}^{x_{E}}=g^{x_{A} x_{E}}=X_{E}^{x_{A}}$ противнику известно.

4. Легитимная сторона $A$ пересылает противнику значение $T_{A}=H\left(I D_{A}\left\|I D_{B}\right\| X_{A}\left\|X_{E}\right\| K_{A} \| 1\right)$, где противнику неизвестен только ключ.

После такого интерактивного взаимодействия противник может проверить правильность предполагаемого пароля $P W^{\prime} \in \mathcal{D}$, вычислив

$$
\begin{aligned}
K_{A}^{\prime} & =H\left(X_{E}^{x_{A}} \| P W^{\prime}\right), \\
T_{A}^{\prime} & =H\left(I D_{A}\left\|I D_{B}\right\| X_{A}\left\|X_{E}\right\| K_{A}^{\prime} \| 1\right)
\end{aligned}
$$

и сравнив вычисленное $T_{A}^{\prime}$ с $T_{A}$, полученным от легитимного участника. Следовательно, противник получает возможность осуществления offlineперебора паролей.

Выделим особенность конструкции этого протокола, приводящую к уязвимости. Описанный метод применим, так как ключ, для которого, строго говоря, выполнено свойство неявной аутентификации, является функцией от неаутентифицированной величины и пароля. Действительно, промежуточное значение $X_{E}^{x_{A}}=g^{x_{A} x_{E}}$ является результатом выполнения протокола Диффи - Хеллмана, уязвимого относительно активного противника ввиду отсутствия механизмов аутентификации. Поэтому при генерации ключа $K_{A}$ противнику неизвестен лишь пароль.

Идею, предложенную в [9], можно считать подходом к устранению указанной уязвимости. Идея состоит в том, чтобы передавать параметры, необходимые для выработки общего ключа, зашифрованными с помощью пароля (из-за этого протоколы семейства $P A K E$ также иногда относят к протоколам типа $E K E$ «Encrypted Key Exchange»). Как уже упоминалось во введении, эта идея в дальнейшем получила существенное развитие и породила множество обобщений и интерпретаций. Однако ниже главное внимание уделяется ее варианту, лежащему в основе протокола SESPAKE, построенному по протоколу Диффи - Хеллмана. 


\section{2. Выбор открытых параметров}

Теперь рассмотрим «игрушечный» протокол, представленный в табл. 2, в котором открытые ключи Диффи-Хеллмана зашифровываются с помощью обычного наложения маски, зависящей от пароля.

Таблица 2. Уязвимая схема протокола семейства $P A K E$

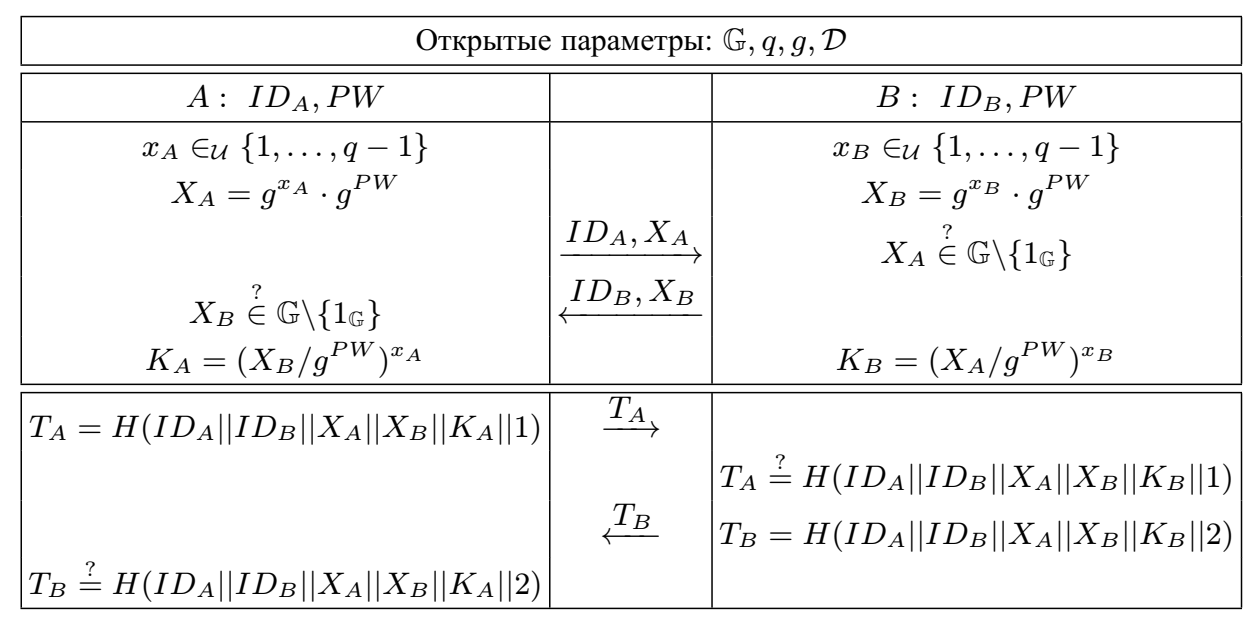

Несмотря на зависимость передаваемых по каналу параметров от пароля, данная схема уязвима относительно угрозы вскрытия пароля. Атака на этот протокол подразумевает противника, который взаимодействует со стороной $A$ от лица стороны $B$ :

1. Легитимная сторона $A$ пересылает противнику сообщение $I D_{A}, X_{A}$.

2. Противник отправляет сообщение $I D_{B}, X_{E}$, в котором $X_{E}=g^{x_{E}}$, $x_{E} \in\{1, \ldots, q-1\}$.

3. На легитимной стороне $A$ вырабатывается ключ

$$
K_{A}=\left(X_{E} / g^{P W}\right)^{x_{A}}=\left(g^{x_{E}-P W}\right)^{x_{A}}=\left(X_{A} / g^{P W}\right)^{x_{E}-P W} .
$$

Заметим, что в этом случае ключ является значением функции только от одного неизвестного противнику параметра - пароля.

4. Легитимная сторона $A$ пересылает противнику значение

$$
T_{A}=H\left(I D_{A}\left\|I D_{B}\right\| X_{A}\left\|X_{E}\right\| K_{A} \| 1\right),
$$

где противнику неизвестен только ключ.

Аналогичным атаке из предыдущего раздела образом противник получает критерий для отбраковки неверных паролей. 
Причины возникновения данной уязвимости в чем-то схожи с теми, которые обсуждались в предыдущем разделе - сессионный ключ удается выразить в виде функции от неаутентифицированной величины, открыто передаваемых параметров и пароля. Однако эта зависимость уже не так тривиальна: для значения ключа, вырабатываемого на стороне $A$, справедливо соотношение

$$
\begin{aligned}
K_{A}=\left(X_{B} / g^{P W}\right)^{x_{A}}=\left(g^{x_{B}} \cdot g^{P W^{\prime}}\right)^{x_{A}} \cdot\left(g^{-P W}\right)^{x_{A}}= \\
=g^{x_{A} x_{B}} \cdot\left(g^{x_{A}}\right)^{P W^{\prime}-P W}=g^{x_{A} x_{B}} \cdot \underbrace{\left(X_{A} / g^{P W}\right)^{P W^{\prime}-P W}}_{f\left(X_{A}, P W\right)} .
\end{aligned}
$$

Здесь значение $g^{x_{A} x_{B}}$ является неаутентифицированным, а функция $f(\cdot, \cdot)$ зависит только от открытого параметра и пароля. Возможность такого представления ключа является следствием того, что для генерации значений $g^{x_{A}}$ и $g^{P W}$ используется один и тот же порождающий элемент $g$. Более того, ключ удается выразить в виде функции от неаутентифицированной величины и перебираемых параметров даже в случае разных порождающих элементов при условии, что дискретные логарифмы одного относительно другого известны. Данный метод вскрытия пароля не будет применим, если в рассмотренном протоколе использовать такие порождающие элементы $g$ и $h$, что дискретные логарифмы $\log _{g} h$ и $\log _{h} g$ неизвестны и трудно вычислимы. Выполнения такого условия можно достичь, если генерировать порождающие элементы случайно и независимо. Однако открытые параметры генерируются разработчиками перед эксплуатацией протокола и публикуются в качестве рекомендуемых, поэтому с целью обеспечения гарантии отсутствия закладок в протоколе разработчики должны доказать, что порождающие элементы действительно выработаны случайно и независимо. Такое доказательство может быть проведено, если генерировать порождающие элементы в соответствии с принципом доказуемой псевдослучайности [2]. Конкретный алгоритм подробно описан в разделе 5.2 .

Еще одним важным аспектом при выборе открытых параметров является структура циклической группы, а именно наличие в ней подгрупп малого порядка. Для примера рассмотрим протокол DragonFly [10] (см. табл. 3), атака на который была предложена в [11].

Первая версия протокола позволяла использовать мультипликативную группу $\mathbb{Z}_{p}^{*}$, где $p$ - любое простое число. В данном случае $\mathbb{G}-$ подгруппа простого порядка $q$ группы $\mathbb{Z}_{p}^{*}$. Пусть в группе $\mathbb{Z}_{p}^{*}$ есть еще одна подгруппа $\mathbb{S}_{r}$ малого порядка $r$. 
Таблица 3. Протокол DragonFly

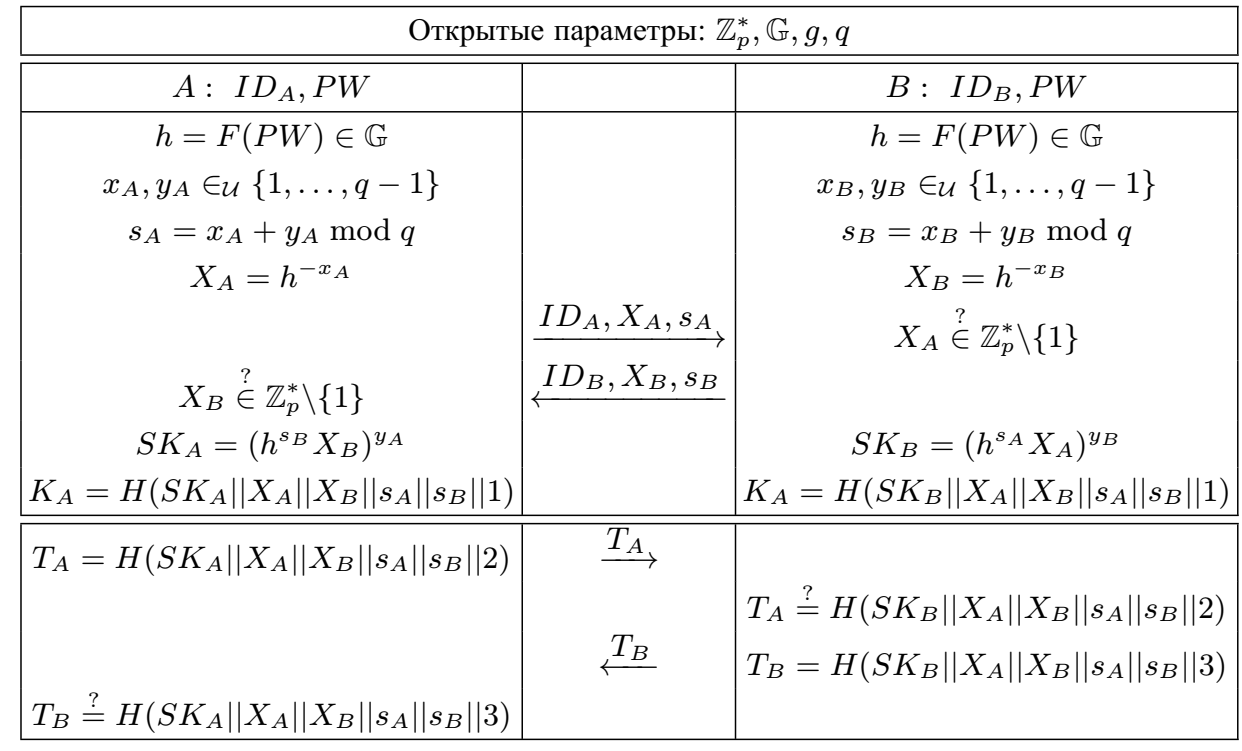

В ходе атаки противник подменяет собой легитимную сторону $B$ :

1. Легитимная сторона $A$ пересылает противнику сообщение $I D_{A}, X_{A}, s_{A}$.

2. Противник отвечает сообщением $I D_{B}, X_{E}, s_{E}$, где $X_{E} \in \mathbb{S}_{r}, s_{E} \in$ $\{1, \ldots, q-1\}$.

3. На легитимной стороне $A$ элемент $X_{E}$ успешно проходит проверку условия принадлежности группе $\mathbb{Z}_{p}^{*}$, после чего вычисляется значение

$$
S K_{A}=\left(h^{s_{E}} X_{E}\right)^{y_{A}}=\left(h^{y_{A}}\right)^{s_{E}} \cdot \underbrace{=}_{\stackrel{D e f}{=}_{E}^{X_{E}^{y_{A}}}}\left(h^{s_{A}} X_{A}\right)^{s_{E}} \cdot X .
$$

4. На этапе аутентификации легитимная сторона $A$ пересылает противнику значение $T_{A}=H\left(S K_{A}\left\|X_{A}\right\| X_{E}\left\|s_{A}\right\| s_{E} \| 2\right)$, где неизвестным параметром является только значение $S K_{A}$.

Заметим, что $S K_{A}=\left(h^{s_{A}} X_{A}\right)^{s_{E}} \cdot X$ является значением функции, неизвестными параметрами которой являются порождающий элемент $h$, зависящий только от пароля, и значение элемента $X$, принадлежащего подгруппе малого порядка. 
Следовательно, противник может вычислять значения $S K_{A}^{\prime}$ и $T_{A}^{\prime}$, перебирая значение пароля $P W^{\prime} \in \mathcal{D}$ и значение элемента $X \in \mathbb{S}_{r}$. Проверка правильности предположения $P W^{\prime}$ осуществляется сравнением $T_{A}^{\prime}$ с полученным ранее $T_{A}$. Таким образом, за одно взаимодействие с легитимными участниками противник получает критерий для отбраковки неверных паролей.

Данная атака не будет применимой, если в схему протокола добавить дополнительные проверки принадлежности элемента подгруппе малого порядка. Такой подход актуален для протоколов, в которых используется, например, группа точек скрученной эллиптической кривой Эдвардса (см., например, [14]), заведомо содержащая подгруппу порядка 4. Другой подход предполагает использование групп, порядок которых является произведением больших близких друг к другу простых чисел.

\section{3. Этап выработки общего ключа}

Помимо свойства неявной аутентификации, подробно описанного в разделе 4.1, этап выработки общего ключа должен обладать некоторыми дополнительными структурными особенностями, усиливающими стойкость протокола. Рассмотрим так называемую атаку со связанными ключами, предложенную в [1], на схему без этапа подтверждения ключа, представленную в табл. 4.

Таблица 4. Уязвимая схема протокола семейства $P A K E$

\begin{tabular}{|c|c|c|}
\hline \multicolumn{3}{|c|}{ Открытые параметры: $\mathbb{G}, q, g, h, \mathcal{D}$} \\
\hline \hline$A: I D_{A}, P W$ & & $B: I D_{B}, P W$ \\
\hline$x_{A} \in \mathcal{U}\{1, \ldots, q-1\}$ & & $x_{B} \in \mathcal{U}\{1, \ldots, q-1\}$ \\
$X_{A}=g^{x_{A}} \cdot h^{P W}$ & & $X_{B}=g^{x_{B}} \cdot h^{P W}$ \\
& $\stackrel{I D_{A}, X_{A}}{\longrightarrow}$ & $X_{A} \stackrel{?}{\in} \mathbb{G} \backslash\left\{1_{\mathbb{G}}\right\}$ \\
$X_{B} \stackrel{?}{\in} \mathbb{G} \backslash\left\{1_{\mathbb{G}}\right\}$ & $\stackrel{I D_{B}, X_{B}}{ }$ & \\
$K_{A}=\left(X_{B} / g^{P W}\right)^{x_{A}}$ & & $K_{B}=\left(X_{A} / g^{P W}\right)^{x_{B}}$ \\
\hline
\end{tabular}

Атака подразумевает активное взаимодействие противника с обеими легитимными сторонами и то, что после выполнения протокола противнику становятся известны ключи, выработанные сторонами $A$ и $B$.

Противник действует следующим образом: 
1. Противник перехватывает сообщение $I D_{A}, X_{A}$ легитимного участника $A$ и вместо него отправляет сообщение $I D_{A}, X_{E}$, где $X_{E}=X_{A}$. $g^{x_{E}}, x_{E} \in\{1, \ldots, q-1\}$.

2. Противник транслирует сообщение $I D_{B}, X_{B}$ легитимного участника $B$ без изменений.

3. Сторона $A$ вырабатывает ключ $K_{A}=\left(X_{B} / h^{P W}\right)^{x_{A}}$.

4. Сторона $B$ вырабатывает ключ

$$
\begin{aligned}
K_{B}=\left(X_{A} \cdot g^{x_{E}} / h^{P W}\right)^{x_{B}}= & \left(X_{A} / h^{P W}\right)^{x_{B}} \cdot g^{x_{E} x_{B}}= \\
& =K_{A} \cdot\left(g^{x_{B}}\right)^{x_{E}}=K_{A} \cdot\left(X_{B} / h^{P W}\right)^{x_{E}} .
\end{aligned}
$$

При известных значениях ключей $K_{A}$ и $K_{B}$ противник получает критерий для перебора паролей без взаимодействия с легитимными участниками, основанный на проверке равенства $K_{B}=K_{A} \cdot\left(X_{B} / h^{P W^{\prime}}\right)^{x_{E}}$ для предполагаемого пароля $P W^{\prime} \in \mathcal{D}$.

Защититься от такой атаки можно с помощью дополнительного применения хэш-функции $H$ к ключу (см. табл. 5).

Таблица 5. Протокол семейства $P A K E$, стойкий против атаки со связанными ключами

\begin{tabular}{|c|c|c|}
\hline \multicolumn{3}{|c|}{ Открытые параметры: $\mathbb{G}, q, g, h, \mathcal{D}$} \\
\hline \hline$A: I D_{A}, P W$ & & $B: I D_{B}, P W$ \\
\hline$x_{A} \in \mathcal{U}\{1, \ldots, q-1\}$ & & $x_{B} \in \mathcal{U}\{1, \ldots, q-1\}$ \\
$X_{A}=g^{x_{A}} \cdot h^{P W}$ & & $X_{B}=g^{x_{B}} \cdot h^{P W}$ \\
& $\stackrel{I D_{A}, X_{A}}{I D_{B}, X_{B}}$ & $X_{A} \stackrel{?}{\in} \mathbb{G} \backslash\left\{1_{\mathbb{G}}\right\}$ \\
$X_{B} \stackrel{?}{\in} \mathbb{G} \backslash\left\{1_{\mathbb{G}}\right\}$ & $\stackrel{ }{\longleftarrow}$ & $K_{B}=H\left(\left(X_{A} / g^{P W}\right)^{x_{B}}\right)$ \\
$K_{A}=H\left(\left(X_{B} / g^{P W}\right)^{x_{A}}\right)$ & &
\end{tabular}

\section{4. Этап подтверждения ключа}

Хотя этап выработки ключа уже предполагает наличие свойства неявной аутентификации, большинство протоколов типа PAKE содержат также этап подтверждения ключа. Однако, будучи некачественно разработанным, данный этап может позволить противнику нарушить явную аутентификацию ключа. Для наглядности рассмотрим так называемые атаки отражения [19], которые могут быть применимы к протоколам согласования ключа с аутентификацией не только на основе пароля и поэтому, вообще говоря, не являются специфичными для протоколов семейства $P A K E$. 
Атака на протокол $P A C E$ [13], схема которого представлена в табл. 6, не позволяет выведать ключ или пароль, но позволяет противнику, который подменяет собой сторону $B$, успешно аутентифицироваться на стороне $A$.

Таблица 6. Протокол РАСЕ

\begin{tabular}{|c|c|c|}
\hline \multicolumn{3}{|c|}{ Открытые параметры: $\mathbb{G}, g, q$} \\
\hline$A: I D_{A}, P W$ & & $B: I D_{B}, P W$ \\
\hline$K_{P W}=H(P W \| 0)$ & & $K_{P W}=H(P W \| 0)$ \\
\hline$s, x_{A}, y_{A} \in_{\mathcal{U}}\{1, \ldots, q-1\}$ & & $x_{B}, y_{B} \in_{\mathcal{U}}\{1, \ldots, q-1\}$ \\
\hline$z=\mathcal{E}_{K_{P W}}(s)$ & $\stackrel{z}{\longrightarrow}$ & $s=\mathcal{E}_{K_{P W}}^{-1}(z)$ \\
\hline$Y_{A}=g^{y_{A}}$ & & $Y_{B}=g^{y_{B}}$ \\
\hline & $\stackrel{Y_{A}}{\longrightarrow}$ & $Y_{A} \stackrel{?}{\in} \mathbb{G} \backslash\left\{1_{\mathbb{G}}\right\}$ \\
\hline$Y_{B} \stackrel{?}{\in} \mathbb{G} \backslash\left\{1_{\mathbb{G}}\right\}$ & $\stackrel{Y_{B}}{\longleftarrow}$ & \\
\hline$h_{A}=g^{s} \cdot Y_{B}^{y_{A}}$ & & $h_{B}=g^{s} \cdot Y_{A}^{y_{B}}$ \\
\hline$X_{A}=h_{A}^{x_{A}}$ & $X_{4}$ & $X_{B}=h_{B}^{x_{B}}$ \\
\hline$?$ & $\underset{X_{B}}{\longrightarrow}$ & $X_{A} \stackrel{\bullet}{\in} \mathbb{G} \backslash\left\{1_{\mathbb{G}}\right\}$ \\
\hline$X_{B} \dot{\in} \mathbb{G} \backslash\left\{1_{\mathbb{G}}\right\}$ & & \\
\hline$S K_{A}=X_{B}^{x_{A}}$ & & $S K_{B}=X_{A}^{x_{B}}$ \\
\hline$K_{A}=H\left(S K_{A} \| 1\right), K_{A}^{\prime}=H\left(S K_{A} \| 2\right)$ & & $K_{B}=H\left(S K_{B} \| 1\right), K_{B}^{\prime}=H\left(S K_{B} \| 2\right)$ \\
\hline$T_{A}=M A C_{K^{\prime}}\left(X_{B} \| h_{A}\right)$ & $\stackrel{\overrightarrow{T_{A}}}{\longrightarrow}$ & \\
\hline & & $T_{A} \stackrel{?}{=} M A C_{K_{B}^{\prime}}\left(X_{B} \| h_{B}\right)$ \\
\hline$T_{B} \stackrel{?}{=} M A C_{K^{\prime}}\left(X_{A} \| h_{A}\right)$ & $\overleftrightarrow{T_{B}}$ & $T_{B}=M A C_{K_{B}^{\prime}}\left(X_{A} \| h_{B}\right)$ \\
\hline
\end{tabular}

1. Легитимная сторона $A$ посылает противнику сообщения $z, Y_{A}$.

2. Противник пересылает стороне $A$ произвольное сообщение $Y_{E} \in$ $\mathbb{G} \backslash\left\{1_{\mathbb{G}}\right\}$.

3. Сторона $A$ пересылает сообщения $X_{A}$ и $T_{A}$, на которые противник отвечает теми же сообщениями $X_{A}$ и $T_{A}$ соответственно.

Заметим, что значение $T_{A}$, пересылаемое противником легитимной стороне $A$, является корректным, так как входные параметры для функции генерации аутентификационной информации для легитимной стороны и для противника одинаковы. Таким образом, противник успешно аутентифицируется на стороне $A$. 
Данный протокол уязвим к рассмотренной атаке, так как он симметричен и генерация аутентификационной информации на обеих сторонах происходит идентичным образом.

Следующая атака, эксплуатирующая уязвимость этапа подтверждения ключа протокола $S P E K E$ [12] (см. табл. 7), предложена в [15]. Эта атака применима, если несколько сеансов протокола для двух легитимных участников могут быть активными одновременно.

Таблица 7. Протокол $S P E K E$

\begin{tabular}{|c|c|c|}
\hline \multicolumn{3}{|c|}{ Открытые параметры: $\mathbb{G}, g, q$} \\
\hline \hline$A: I D_{A}, P W$ & & $B: I D_{B}, P W$ \\
\hline$h=F(P W) \in \mathbb{G}$ & & $h=F(P W) \in \mathbb{G}$ \\
$x_{A} \in \mathcal{U}\{1, \ldots, q-1\}$ & & $x_{B} \in \mathcal{U}\{1, \ldots, q-1\}$ \\
$X_{A}=h^{x_{A}}$ & & $X_{B}=h^{x_{B}}$ \\
& $\frac{I D_{A}, X_{A}}{\longrightarrow}$ & $X_{A} \stackrel{?}{\in} \mathbb{G} \backslash\left\{1_{\mathbb{G}}\right\}$ \\
$X_{B} \stackrel{?}{\in} \mathbb{G} \backslash\left\{1_{\mathbb{G}}\right\}$ & $\stackrel{I D_{B}, X_{B}}{ }$ & \\
$K_{A}=H\left(X_{B}^{x_{A}}\right)$ & & $K_{B}=H\left(X_{A}^{x_{B}}\right)$ \\
\hline \hline$T_{A}=H\left(H\left(K_{A}\right)\right)$ & $\stackrel{T_{A}}{\longrightarrow}$ & \\
& & $T_{A} \stackrel{?}{=} H\left(H\left(K_{B}\right)\right)$ \\
$T_{B} \stackrel{?}{=} H\left(K_{A}\right)$ & $\stackrel{T_{B}}{\longleftarrow}$ & $T_{B}=H\left(K_{B}\right)$ \\
\hline
\end{tabular}

Противник взаимодействует с легитимным участником $A$, который сам является инициатором соединения в соответствии с протоколом. Также противник может первым инициировать соединение. В результате атаки противник успешно аутентифицируется на стороне $A$ от лица стороны $B$.

Действия противника на этапе выработки ключа:

1. Легитимная сторона $A$ инициирует первое соединение, пересылая противнику значение $I D_{A}, X_{A}$.

2. Противник инициирует второе соединение, посылая легитимной стороне $A$ сообщение $I D_{B}, X_{A}$.

3. Сторона $A$ в процессе второго соединения пересылает значение $I D_{A}, X_{A}^{\prime}$.

4. Противник для первого соединения отвечает сообщением $I D_{B}, X_{A}^{\prime}$. 
Действия противника на этапе явной аутентификации:

1. Легитимная сторона $A$ в процессе первого соединения пересылает противнику значение $T_{A}$.

2. Противник для второго соединения посылает $A$ сообщение $T_{A}$.

3. Сторона $A$ в процессе этого же соединения пересылает значение $T_{A}^{\prime}$.

4. Противник для первого соединения отвечает сообщением $T_{A}^{\prime}$.

Данная атака применима, так как на этапе явной аутентификации значение $T_{A}$ не зависит от идентификатора участника, генерирующего это значение.

\section{5. Формат представления данных}

Совместное использование методов криптографии с секретным и открытым ключами требует наличия преобразований битовых строк в некоторые, зачастую нетривиальные, группы и обратно (например, в группы точек эллиптической кривой). Такие преобразования кодирующего характера обычно называют форматами представления данных. Использование при этом малоэнтропийного секрета накладывает жесткие ограничения на некоторые из них. Рассмотрим пример схемы, использующей группу точек эллиптической кривой (см. табл. 8).

Таблица 8. Уязвимый протокол типа $P A K E$

\begin{tabular}{|c|c|c|}
\hline \multicolumn{3}{|c|}{ Открытые параметры: $\mathbb{E}, P, q, \mathcal{D}$} \\
\hline \hline$A: I D_{A}, P W$ & & $B: I D_{B}, P W$ \\
\hline$K_{P W}=H(P W)$ & & $K_{P W}=H(P W)$ \\
$x_{A} \in \mathcal{U}\{1, \ldots, q-1\}$ & & $x_{B} \in \mathcal{U}\{1, \ldots, q-1\}$ \\
$X_{A}=x_{A} \cdot P$ & & $X_{B}=x_{B} \cdot P$ \\
$Y=\mathcal{E}_{K_{P W}}\left(X_{A}\right)$ & $\stackrel{Y}{\longrightarrow}$ & $X_{A}=\mathcal{E}_{K_{P W}}^{-1}(Y)$ \\
& & $X_{A} \stackrel{?}{\in} \mathbb{E} \backslash\left\{0_{\mathbb{E}}\right\}$ \\
$X_{B} \stackrel{?}{\in} \mathbb{E} \backslash\left\{0_{\mathbb{E}}\right\}$ & $\stackrel{X_{B}}{ }$ & \\
$K_{A}=H\left(x_{A} \cdot X_{B} \| 0\right)$ & & $K_{B}=H\left(x_{B} \cdot X_{A} \| 0\right)$ \\
\hline \hline$T_{A}=H\left(Y\left\|X_{B}\right\| K_{A} \| 1\right)$ & $\stackrel{T_{A}}{\longrightarrow}$ & \\
& $T_{A} \stackrel{?}{=} H\left(Y\left\|X_{B}\right\| K_{B} \| 1\right)$ \\
$T_{B} \stackrel{?}{=} H\left(Y\left\|X_{B}\right\| K_{A} \| 2\right)$ & $\stackrel{T}{T_{B}=H\left(Y\left\|X_{B}\right\| K_{B} \| 2\right)}$ \\
\hline
\end{tabular}


Стойкость этой схемы существенным образом зависит от используемого формата представления точки кривой. Пусть в процессе шифрования точки $X_{A}=(x, y)$ используется ее каноническое представление в форме Вейерштрасса в виде двоичной строки $x \| y$ (конкатенации битовых представлений значений $x$ и $y$ ). В этом случае схема уязвима относительно вскрытия пароля даже при присутствии в канале противника, только прослушивающего канал, - самого слабого из возможных противников.

Противник, получив $Y$, расшифровывает его с помощью ключа $K_{P W^{\prime}}=$ $H\left(P W^{\prime}\right)$, перебирая значения $P W^{\prime} \in \mathcal{D}$. При правильном пароле результат расшифрования $x^{\prime}|| y^{\prime}$ должен удовлетворять уравнению кривой Вейерштрасca, т.е. $x^{\prime}$ определяет почти все биты $y^{\prime}$. Если же пароль не верен, то с вероятностью, близкой к единице, $x^{\prime}$ и $y^{\prime}$ не будут удовлетворять указанному уравнению. На этом основан критерий для проверки истинности паролей: если $X_{A}^{\prime}=\left(x^{\prime}, y^{\prime}\right) \notin \mathbb{E}$, то пароль $P W^{\prime}$ точно не является истинным. Таким образом, противник получает критерий истинности пароля, позволяющий ему организовывать offline-перебор.

Протокол будет неуязвим к этой атаке, если использовать краткое представление точки кривой в форме Вейерштрасса (значение абсциссы $x$ и один бит, отвечающий за знак значения ординаты $y$ ).

Рассмотренная атака является частным случаем атак на протоколы, в которых структурированные данные шифруются на ключе, зависящем только от пароля (см. [9]).

\section{5. Протокол SESPAKE}

В данном разделе приводится табличное описание протокола SESPAKE, полное описание которого с учетом технических методов защиты приведено в документе [16] (см. также [3]). С использованием рассмотренных выше уязвимостей объясняются различные особенности строения этого протокола.

\section{1. Описание протокола}

Протокол SESPAKE в качестве группы использует группу точек эллиптической кривой, удовлетворяющей требованиям документа [18]. Клиент и сервер хранят $l$ точек $Q_{1}, \ldots, Q_{l}$ и точку $P$. Сервер дополнительно хранит следующие параметры, из которых в секрете необходимо держать лишь $Q_{P W}$ :

- число ind $\in\{1, \ldots, l\}$;

- строка salt;

- точку $Q_{P W}=\operatorname{int}(f(P W$, salt, 2000$)) \cdot Q_{i n d}$, где $f$ - функция PBKDF2 [17], int - инъективное отображение $V_{n}$ в $\left\{0,1, \ldots, 2^{n}-1\right\}, n$ - константа, соответствующая используемой кривой. 
Клиент дополнительно хранит пароль $P W$, который также необходимо держать в секрете. Параметр $l$ может быть выбран произвольным натуральным числом. Схема протокола приведена в табл. 9.

Таблица 9. Протокол SESPAKE

\begin{tabular}{|c|c|c|c|}
\hline 4 & \multicolumn{3}{|c|}{ Открытые параметры: $\mathbb{E}, P, Q_{1}, \ldots, Q_{l}, m, q, \mathcal{D}$} \\
\hline & $A: I D_{A}, P W$ & & $B: I D_{B}, Q_{P W}$, ind, salt \\
\hline $\begin{array}{l}2 \\
3\end{array}$ & $\begin{array}{c}z_{A}=0 \\
Q_{P W}^{A}= \\
=i n t(f(P W, \text { salt }, 2000)) \cdot Q_{i n d} \\
x_{A} \in \mathcal{U}\{1, \ldots, q-1\} \\
X_{A}=x_{A} \cdot P-Q_{P W}^{A} \\
\\
X_{B} \stackrel{?}{\in} \mathbb{E} \\
Q_{A}=X_{B}-Q_{P W}^{A} \\
\frac{m}{q} Q_{A} \neq 0_{\mathbb{E}} \Rightarrow \\
Q_{A}=x_{A} P, z_{A}=1 \\
K_{A}=H\left(\left(\frac{m}{q} \cdot x_{A} \bmod q\right) Q_{A}\right)\end{array}$ & $\stackrel{\stackrel{I D_{B}}{\longrightarrow}}{\stackrel{I D_{A}, \text { ind, salt }}{\longleftarrow}}$ & $\begin{array}{c}X_{A} \stackrel{?}{\in} \mathbb{E} \\
Q_{B}=X_{A}+Q_{P W} \\
\text { if } \frac{m}{q} Q_{B} \neq 0_{\mathbb{E}} \Rightarrow \\
\Rightarrow Q_{B}=x_{B} P, z_{B}=1 \\
x_{B} \in \mathcal{U}\{1, \ldots, q-1\} \\
K_{B}=H\left(\left(\frac{m}{q} \cdot x_{B} \bmod q\right) Q_{B}\right) 1 \\
X_{B}=x_{B} \cdot P+Q_{P W}\end{array}$ \\
\hline & \multicolumn{3}{|c|}{$\begin{aligned} \operatorname{tag}_{A} & =I D_{A} \| \text { ind } \| \text { salt }\left\|X_{A}\right\| X_{B} \\
\operatorname{tag}_{B} & =I D_{B} \| \text { ind } \| \text { salt }|| X_{A} \| X_{B}\end{aligned}$} \\
\hline 5 & $\begin{array}{c}T_{A}=M A C_{K_{A}}\left(\operatorname{tag}_{A}\right) \\
T_{B} \stackrel{?}{=} M A C_{K_{A}}\left(\operatorname{tag}_{B}\right) \& z_{A} \stackrel{?}{=} 0\end{array}$ & $\begin{array}{l}\stackrel{T_{A}}{\longrightarrow} \\
\stackrel{T_{B}}{\longleftarrow}\end{array}$ & $\begin{array}{c}T_{A} \stackrel{?}{=} M A C_{K_{B}}\left(\operatorname{tag}_{A}\right) \& z_{B} \stackrel{?}{=} 0 \\
T_{B}=M A C_{K_{B}}\left(\operatorname{tag}_{B}\right)\end{array}$ \\
\hline
\end{tabular}




\section{2. Принципы построения протокола $S E S P A K E$}

Протокол SESPAKE спроектирован с учетом рассмотренных выше уязвимостей в соответствии с подходами, предлагаемыми для их устранения. Комментарии к меткам в первом столбце табл. 9, указывающим на реализацию этих подходов в протоколе, приведены ниже:

1. Для неприменимости атаки с использованием подгруппы малого порядка (см. с. 15) проводится проверка точек вида

$$
\frac{m}{q} Q_{B} \stackrel{?}{=} 0_{\mathbb{E}} .
$$

При определенной структуре множества парольных точек обрыв соединения непосредственно после неуспеха данной проверки может привести к увеличению вероятности угадывания пароля противником при активном взаимодействии. Поэтому желательно использовать дополнительные меры ( $\left.Q_{B}=x_{B} P, z_{B}=1\right)$, которые не позволяют детектировать случай возникновения точки малого порядка. Присваивание $Q_{B}=x_{B} P$ является примером того, как избежать уменьшения времени вычисления значения $K_{A}$. Возникающая разница во времени могла бы использоваться для детектирования данного случая.

2. Использование хэш-функции необходимо для защиты против атаки со связанными ключами (см. раздел 4.3).

3. Несимметричность протокола, заключающаяся в наличии разных операций для участников ( $-Q_{P W}^{A}$ для участника $A,+Q_{P W}$ для участника $\left.B\right)$, делает неприменимой атаку отражения (см. раздел 4.4).

4. Для того чтобы протокол был неуязвим к атаке, описанной в разделе 4.2, кратности любой точки из набора $Q_{1}, \ldots, Q_{l}$ относительно порождающего элемента $P$ и относительно других точек должны быть неизвестными (см. раздел 4.2). Причем задача вычисления этой кратности должна быть неосуществима на практике, т. е. ее трудоемкость должна быть сравнима с трудоемкостью решения сложной задачи дискретного логарифмирования в группе точек используемой эллиптической кривой.

Выбор точки эллиптической кривой, заданной в форме Вейерштрасса $\left(y^{2}=x^{3}+a x+b\right)$, в соответствии с принципом доказуемой псевдослучайности может быть осуществлен следующим образом: 
I. Сгенерировать случайную строку $s$.

II. Положить $x=H(s)$.

III. Если значение $x^{3}+a x+b$ не является квадратичным вычетом по модулю $p$, перейти к первому пункту.

IV. Положить $y=\sqrt{x^{3}+a x+b}(\bmod p)$.

Здесь $p-$ порядок поля, над которым строится группа точек эллиптической кривой.

Доказательством случайности и независимости полученных порождающих элементов является предоставление прообразов их абсцисс относительно хэш-функции $H$. Действительно, если хэш-функция является криптографически стойкой, то задача поиска порождающих элементов с известными взаимными кратностями и нахождения прообразов их абсцисс является вычислительно сложной.

5. Использование идентификаторов $I D_{A}$ и $I D_{B}$ при вычислении значений $T_{A}, T_{B}$ на этапе явной аутентификации защищает от атаки отражения для параллельных сессий (см. с. 20).

\section{3. Защита от online-перебора и ее следствие}

Пароль - величина малоэнтропийная, поэтому в отличие от длинного ключа нельзя пренебрегать вероятностью простого угадывания этого пароля при попытках установить соединение в соответствии с протоколом (onlineперебор).

Значит, использование паролей в качестве общего предварительно согласованного секрета должно сопровождаться такими техническими мерами, как ограничение на количество попыток установить соединение или введение временных задержек между неуспешными попытками.

Однако использование таких контрмер приводит к другой потенциальной опасности, а именно к возможности DDoS-атаки. Хотя для борьбы с атаками данного типа предложено множество методов, при установлении параметров счетчиков необходимо соблюдать баланс между вероятностями осуществления угроз online-перебора и DDoS-атаки. 


\section{6. Заключение}

Рассмотрение конкретных атак и применение известных методов защиты от них не гарантируют, что разрабатываемый протокол будет стойким, так как нельзя учесть все возможные, но пока не известные атаки. Формулировка эффективного конструктивного критерия, позволяющего строить неуязвимые протоколы, представляется сложной исследовательской задачей.

Единственным известным авторам подходом к ее решению является использование методов математической криптографии, которые позволяют получать оценки стойкости протоколов в определенных моделях безопасности. Наиболее популярной моделью противника для протоколов типа PAKE является модель противника на основе неотличимости (см. [8]), в которой формулируется задача отличения вырабатываемого сессионного ключа от случайной строки. Доказательство стойкости в такой модели предполагает сведение упомянутой выше задачи к некоторой задаче, считающейся сложной, например к задаче дискретного логарифмирования (см., например, [1]). Неформально говоря, такое сведение показывает, что самой лучшей тактикой для противника является опробование пароля в ходе попытки установить соединение, честно следуя протоколу.

Для протокола SESPAKE обоснование стойкости удается провести как в указанной модели с угрозой отличения ключа от случайной строки, так и в модели с угрозой ложной аутентификации. Описание полученных оценок и их доказательств планируется привести в будущих статьях.

\section{Список литературы}

[1] Abdalla M., Pointcheval D., "Simple password-based encrypted key exchange protocols". In: "Proc. of Topics in Cryptology - CT-RSA 2005", Lect. Notes Comput. Sci., 3376, 191-208.

[2] Lochter M., Merkle J., Schmidt J.-M., Schutze T., "Requirements for standard elliptic curves", Cryptology ePrint Archive, Report 2014/832.

[3] Smyshlyaev S. (Ed.), Alekseev E., Oshkin I., Popov V., "CRYPTO-PRO. The security evaluated standardized password authenticated key exchange (SESPAKE) Protocol”, Internet-Draft (2016), https://tools.ietf.org/html/draft-smyshlyaev-sespake-02.

[4] Eronen P., Tschofenig H. (Eds.), "Pre-shared key ciphersuites for transport layer security (TLS)", RFC 4279 (2005), https://tools.ietf.org/html/rfc4279. 
[5] Bellare M., Rogaway P., "Entity authentication and key distribution". In: "CRYPTO'93", Lect. Notes Comput. Sci., 773, 1993, 232-249.

[6] Diffie W., van Oorschot P.C., Wiener M. J., "Authentication and authenticated key exchanges", Designs, Codes and Cryptography, $2: 2$ (June 1992), 107-125.

[7] Needham R. M., Schroeder M. D., "Using encryption for authentication in large networks of computers", Comm. ACM, $21: 12$ (December 1978), 993-999.

[8] Bellare M., Pointcheval D., Rogaway P., "Authenticated key exchange secure against dictionary attacks". In: “EUROCRYPT 2000”, Lect. Notes Comput. Sci., 1807, 2000, 139-155.

[9] Bellovin S., Merritt M., "Encrypted key exchange: password-based protocols secure against dictionary attacks". In: "IEEE Symposium on Security and Privacy", 1992, 72-84.

[10] Harkins D., "Simultaneous authentication of equals: A secure, password-based key exchange for mesh networks". In: "Proceedinigs of SENSORCOMM 2008", Washington, DC: IEEE Computer Society, 2008, 839-844.

[11] Clarke D., Hao F., "Cryptanalysis of the Dragonfly key exchange protocol", IET Information Security, 8:6 (2014), 283-289.

[12] Jablon D., "Strong password-only authenticated key exchange", ACM SIGCOMM Computer Communication Review, $26: 5$ (1996), 5-26.

[13] Federal Office for Information Security (BSI): Advanced security mechanism for machine readable travel documents - extended access control (eac), password authenticated connection establishment (PACE), and restricted identification (ri), 2008.

[14] Smyshlyaev S. (Ed.), Alekseev E., Oshkin I., Popov V., Leontiev S. Podobaev V. Belyavsky D., "Guidelines on the Cryptographic Algorithms to Accompany the Usage of Standards GOST R 34.10-2012 and GOST R 34.11-2012"”, RFC 7836 (2016), https://tools.ietf.org/html/rfc7836.html.

[15] Hao F., Shahandashti S. F., “The SPEKE Protocol revisited.”, Cryptology ePrint Archive, Report $2014 / 585$.

[16] Информационная технология. Криптографическая защита информачии. Протокол выработки общего ключа с аутентификацией на основе пароля, Рекомендации по стандартизации, М.: Стандартинформ, 2016.

[17] Информационная технология. Криптографическая защита информации. Парольная защита ключевой информации, Рекомендации по стандартизации, М. : Стандартинформ.

[18] Начиональный стандарт Российской Федерачии ГОСТ Р 34.10-2012 Информационная технология. Криптографическая защита информации. Процессы формирования и проверки электронной иифровой подписи, Федеральное агентство по техническому регулирвоанию и метрологии, М.: Стандартинформ, 2012. 
[19] Menezes A. J., van Oorschot P.C., Vanstone S. A., Handbook of Applied Cryptography, Boca Raton, FL, etc.: CRC Press, 1996, 780 pp. 